\title{
Modificación estructural de la lignina extraída a partir de carbones de bajo rango para la obtención de madera sintética
}

\section{Structural modification of extracted lignin from low rank coals for manufacturation of synthetic woods}

\author{
Jesús Álvaro Jiménez Montoya \\ Químico, Licenciado en Química, Magíster en Química. Profesor Titular de la Universidad \\ Distrital Francisco José de Caldas. Bogotá, Colombia.jajymenez@gmail.com \\ Bibiana Gordillo Díaz \\ Estudiante de Licenciatura en Química de la Universidad Distrital Francisco José de Caldas. \\ Bogotá, Colombia. bibis00@msn.com \\ Marco Antonio Vega Atuesta \\ Estudiante de Licenciatura en Química de la Universidad Distrital Francisco José de Caldas. \\ Bogotá, Colombia. vegatu182@yahoo.com \\ Clasificación del artículo: investigación (Conciencias) \\ Fecha de recepción: mayo 7 de 2010 \\ Fecha de aceptación: noviembre 23 de 2010
}

Palabras clave: Aglomerante, Carbón, Lignina, Madera sintética, Turba.

Key words: Binder, Coal, Lignine, Synthetic wood, Peat.

\section{RESUMEN}

El grupo Investigación en Carbones de la Universidad Distrital Francisco José de Caldas ha trabajado en la extracción de lignina a partir de carbón de bajo rango y de turba, con el fin de emplearla en la elaboración de madera sintética y de esta manera aplicarla a la industria maderera.

En una primera parte se aplicaron los métodos Sosa, Sulfato y Bisulfito [1], modificados para la extracción de lignina a partir de pino y cedro, se evidenció que el método Sosa era el de mayor rendimiento. Este método fue aplicado para la extracción de lig- nina a partir de una muestra de carbón leonardítico. Posteriormente, ésta se utilizó en la elaboración de la resina lignina-fenol-formaldehído, la cual actúa como aglomerante de fibras naturales [2].

La segunda parte se fundamentó en la estandarización del método Sosa [3], en el cual se modificó la presión utilizando autoclave para una muestra de turba, lo cual mejoró el rendimiento. La lignina obtenida se empleó en la elaboración de resinas tales como lignina-fenolformaldehído, lignina-resorcinol-formaldehído y lignina-polipropileno, las cuales fueron utilizadas empleando como fibra natural bagazo de caña. 


\section{con-ciencias}

En la actualidad se está desarrollando una tercera etapa en la que se ha realizado la extracción de lignina por el método Sosa estandarizado para una muestra de turba; esta lignina obtenida se pretende modificar estructuralmente por radiación UV (método físico) y con $\mathrm{NaOH}$ (método químico), con el fin de aumentar su reactividad en la obtención de resinas [4] y mejorar de esta manera la calidad de la madera elaborada.

\section{Abstract}

The Universidad Distrital Francisco José de Caldas Coal Research Group has been working with lignin extraction from peat and low rank coal and using the obtained extract for synthetic wood industry.

Sosa, sulfate and bisulfite methods [1] were applied in the first research step and later they were modified with the lignine extraction process from pine and cedar. Among these, Sosa method showed as the more efficient method, so, it was used to extract lignine from low rank coal. The extracted lignin was used to prepare lignin-phenol-formaldehide resin and it works as natural fiber binder [2].

In the second research step, standarization Sosa method was made [3], were pressure was modified using an pressure reactor (autoclave) for a peat sample. Efficiency was improved. The obtained lignine was used to prepare resins such as phenolformaldehide- lignin, resorcinol-formaldehydelignin and polypropilen-lignin. All of them were utilized using sugar cane bagasse as natural fiber.

Currently, a third research step is being developed using Sosa method and working with a peat sample. The main purpose, is to modificate the extracted lignin structure by UV radiation (physical methot) and with $\mathrm{NaOH}$ (Chemical method) so the lignin reactivity is improved in the resins production [4] and as result, to get a better quality synthetic wood.

\section{Introducción}

Desde hace varios años se ha venido produciendo en todo el planeta un proceso de destrucción del bosque tropical a gran escala, "lo que ha llevado a la creación de políticas que han generado una reducción de la pérdida neta de superficie forestal de 8.3 millones de hectáreas al año en la década de 1990 a 5.2 millones de hectáreas anuales entre 2000 y 2010", como se da a conocer en [5], y aunque ha decrecido la deforestación esta cifra no es significativa, pues si no se reduce más los bosques tropicales irán desapareciendo lo cual generará efectos impredecibles en el clima mundial.

Actualmente se han diseñado alternativas para minimizar la deforestación, basadas en el estudio de opciones que reemplacen la madera en la industria, algunos investigadores han tomado la lignina como objeto de estudio ya que ésta es una macromolécula

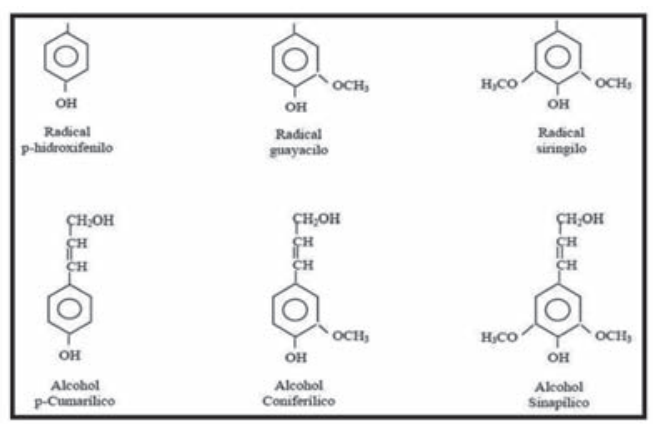

Figura 1. Unidades y monómeros básicos de la lignina [7]. 


\section{con-ciencias}

Se pretende que la lignina influya directamente en la elaboración de productos sintéticos que sustituyan a la madera, tal como se evidencia en el estudio realizado por Martínez J. y cols., "Debido a que el fenol es una materia prima costosa y tóxica derivada de la industria petrolera, se ve la necesidad de buscar alternativas para reemplazarlo en la elaboración de resinas, las cuales son la base de estos productos. Y la lignina representa una buena elección, especialmente en resinas fenólicas obtenidas de la policondensación entre el formaldehído y un exceso de fenol" [8].

No sólo este tipo de resinas han sido modificadas sino también las resinas fenólicas tipo novolaca por medio de la lignina extraída del licor negro del bagazo de caña, según lo da a conocer Martínez J. y cols. [9]. Así mismo se ha utilizado la lignina de licor negro para sustituir las resinas en aglomerados de madera, como lo reporta el grupo de investigación del Dr. Velásquez de la Universidad Pontificia Bolivariana en su trabajo titulado Utilización de lignina de licor negro para la sustitución de resinas en aglomerados de madera [10].

De esta manera cobra importancia para el grupo Investigación en Carbones de la Universidad Distrital Francisco José de Caldas estudiar la extracción de lignina a partir del carbón tipo leonardítico y de la turba, debido al origen vegetal que le proporciona un porcentaje significativo de lignina, su bajo proceso de carbonificación (ver Figura 2) y su abundancia en el país, además de su económico método de explotación. El fin es utilizar la lignina en la elaboración de productos sintéticos maderables.

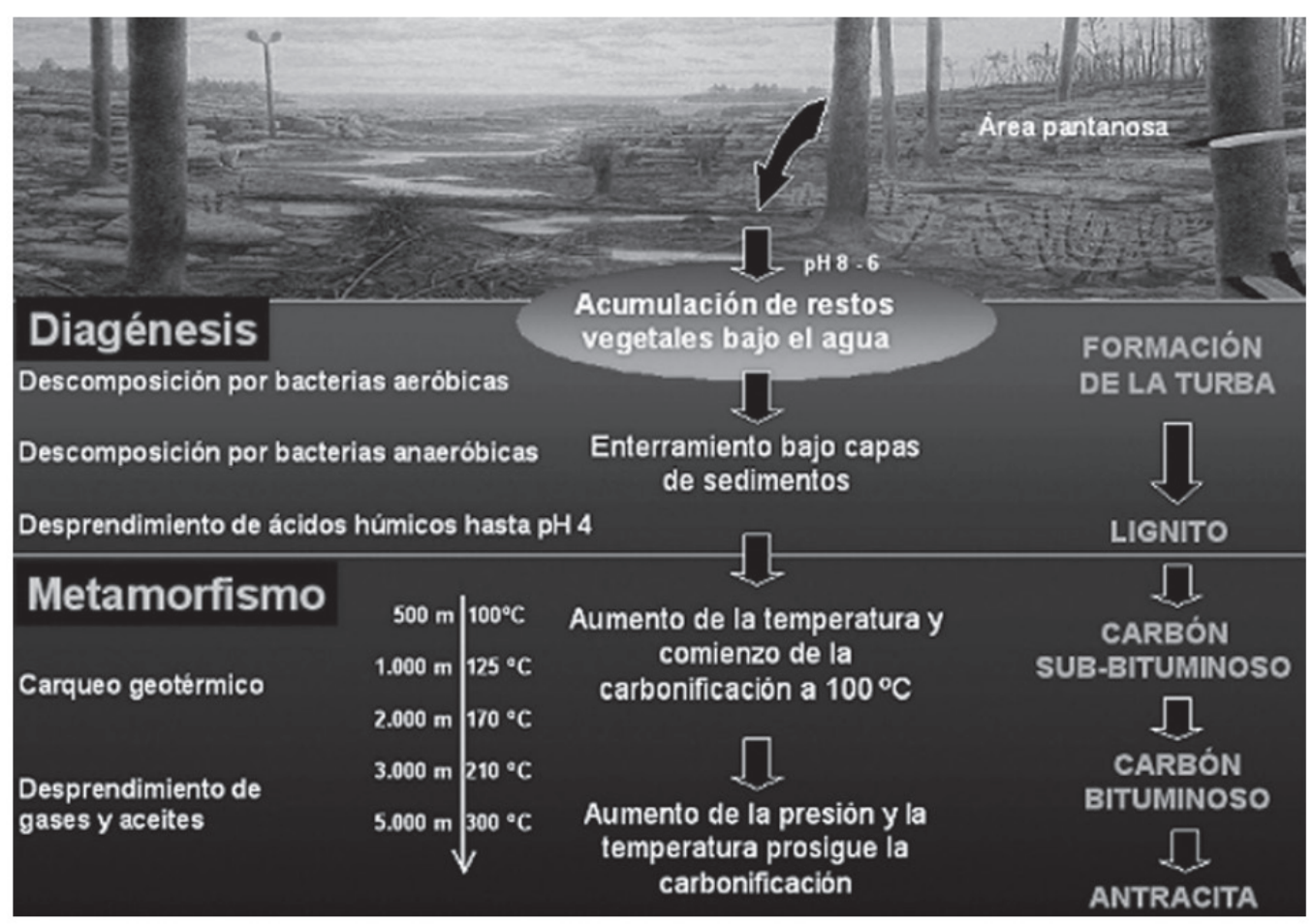

Figura 2. Proceso de carbonificación [11]. 


\section{con-ciencias}

Esta investigación se ha dado en etapas que han fortalecido la metodología propuesta. En la primera etapa se realizó la extracción de lignina por los métodos Sosa, Bisulfito y Sulfato, los cuales se modificaron con el fin de adaptarlos a las condiciones de laboratorio para una muestra de pino y cedro, el método Sosa se evidenció con el mayor rendimiento.

En la segunda etapa se trabajó en la estandarización del método Sosa, modificándolo a condiciones de laboratorio y adaptándolo a una autoclave para simular una presión constante y superior a la del ambiente durante el proceso, y usando una muestra de turba tomada de la zona carbonífera del municipio de Paipa, Boyacá, con un porcentaje mayor de lignina que el carbón de bajo rango.

Tanto en la primera como en la segunda etapa se realizaron ensayos en la elaboración del producto maderable utilizando la lignina extraída, mostrando resultados que motivaron a estructurar una tercera etapa en la que actualmente se está trabajando con una muestra de turba tomada de la misma zona carbonífera. En ésta se pretende extraer la lignina por el método Sosa y modificarla estructuralmente a través de su despolimerización y posterior hidroximetilación, con el fin de aumentar su reactividad en la formación de resinas y el desempeño de éstas como aglomerantes en la unión de fragmentos de una o varias sustancias y dar unidad al conjunto, por efectos exclusivamente físicos [12] en la elaboración de un producto maderable de alta calidad que sea competitivo con la madera a nivel industrial.

\section{Metodología}

\subsection{Primera etapa}

Con el fin de extraer lignina de una muestra de carbón de bajo rango se realizaron pruebas de extracción con métodos propuestos para la madera: el método Sosa, Bisulfito y Sulfato [1], modificados para reflujo, empleando $10 \mathrm{~g}$ de muestra de pino y cedro y 2,5 g de las mismas muestras para el método orgánico Fukushina, también modificado a condiciones de laboratorio.

El método Sosa modificado se trabajó a diferentes concentraciones de álcali, empleando como mezcla extractante $7 \mathrm{ml}$ de $\mathrm{NaOH}(10 \%, 20 \%, 30 \%$ y $40 \% \mathrm{P} / \mathrm{V}$ (Peso/Volumen)) y $50 \mathrm{ml} \mathrm{de} \mathrm{Na}_{2} \mathrm{CO}_{3} 10 \%$ $\mathrm{P} / \mathrm{V}$, durante tiempos de $48 \mathrm{~h}, 72 \mathrm{~h}$ y $95 \mathrm{~h}$. Hasta la temperatura de ebullición, se filtró con el fin de obtener el licor negro para cada caso y se acidificó con $\mathrm{H}_{2} \mathrm{SO}_{4}$ hasta $\mathrm{pH} 2 \mathrm{y}$, posteriormente, se lavó con agua deionizada hasta $\mathrm{pH}$, obteniendo de estos procesos la lignina pura.

El método Bisulfito utilizó como extractante 125 $\mathrm{ml}$ de Bisulfito de sodio $10 \% \mathrm{P} / \mathrm{V}$ hasta ebullición durante un periodo de $9 \mathrm{~h}-10 \mathrm{~h}$ para luego filtrar, obteniendo así el licor negro, el cual se centrifugó y se obtuvo la lignina libre de impurezas después de varios lavados con agua deionizada hasta $\mathrm{pH} 6$.

En el método Sulfato se utilizó una mezcla de 75 $\mathrm{ml} \mathrm{Na} \mathrm{SO}_{4} 10 \% \mathrm{P} / \mathrm{V}$ y $50 \mathrm{ml} \mathrm{Na}_{2} \mathrm{CO}_{3} 10 \% \mathrm{P} / \mathrm{V} \mathrm{du}-$ rante $11 \mathrm{~h}-24 \mathrm{~h}$; hasta ebullición, la mezcla obtenida se filtró, teniendo como filtrado el licor negro de lignina que por último se acidificó con $\mathrm{H}_{2} \mathrm{SO}_{4}$ hasta $\mathrm{pH} 2$ y se lavó con agua deionizada hasta $\mathrm{pH} 6$ para obtener la lignina purificada.

Así mismo, se empleó el método Fukushina modificado usando una mezcla extractante de $\mathrm{HCl} 2$ N (normalidad) y Dioxano $100 \%$ 9:1, en la que se dejó la muestra en reposo durante $48 \mathrm{~h}$. Posteriormente, se filtró realizando lavados con dioxano, se neutralizó con $2 \mathrm{~g}$ de $\mathrm{NaHCO}_{3} \mathrm{y}$ de nuevo se filtró para luego rotaevaporar a $40{ }^{\circ} \mathrm{C}$, en este momento se obtuvo un residuo que a continuación fue agitado magnéticamente y centrifugado y se obtuvo lignina hidratada, por lo cual se llevó a secado por 30 minutos a $55^{\circ} \mathrm{C}$. Por último, con el fin de purificar la lignina, ésta se disolvió en dioxano $100 \%$ y se filtró; el filtrado se mezcló con $100 \mathrm{ml}$ de éter etílico anhídrido, se agitó y se adicionó $40 \mathrm{ml}$ de Petrol; teniendo como resultado final la lignina pura. 


\section{con-ciencias}

$\mathrm{A} \pm 0,1 \mathrm{~g}$ de cada una de las ligninas obtenidas se les realizaron pruebas de solubilidad en $1 \mathrm{ml}$ de cada uno de los siguientes solventes: $\mathrm{NaOH} 10 \%$ $\mathrm{P} / \mathrm{V}, \mathrm{HCl} 10 \% \mathrm{~V} / \mathrm{V}$ (Volumen/Volumen); $\mathrm{H}_{2} \mathrm{SO}_{4}$, EtOH, éter, $\mathrm{MeOH}, \mathrm{CHCl}_{3}, \mathrm{CCl}_{4}, \mathrm{NaHCO}_{3} 10 \%$ $\mathrm{P} / \mathrm{V}$ y $\mathrm{H}_{2} \mathrm{O}$ destilada. Posterior a ello, se procedió al análisis químico inorgánico (determinación de aniones y cationes) y orgánico elemental, al igual que pruebas cualitativas de identificación, tales como la de Maule, Wiesner y anilina en $\mathrm{H}_{2} \mathrm{SO}_{4}$.

En la prueba de Maule se utilizó $1 \mathrm{ml}$ de $\mathrm{NaOH}$ $10 \% \mathrm{P} / \mathrm{V}$ y para $\pm 0,1 \mathrm{~g}$ de las ligninas obtenidas en cada uno de los métodos, con agitación constante, se les adicionó 2-6 gotas de $\mathrm{KMnO}_{4} 1 \% \mathrm{P} / \mathrm{V}$, a esto se le adicionó $1 \mathrm{ml} \mathrm{HCl} \mathrm{1N}$, obteniéndose así una coloración rojo oscuro.

Para la prueba de Wiesner se empleó $1 \mathrm{ml}$ de $\mathrm{HCl}$ $6 \mathrm{~N} \mathrm{y} \pm 0.1 \mathrm{~g}$ de las diferentes ligninas, homogenizando permanentemente y se agregó floroglucina etanólica $1 \% \mathrm{P} / \mathrm{V}$, observándose un color rojo guinda después de 1 minuto de agitación.

Para la prueba de la anilina, se usó $1 \mathrm{ml}$ de esta pura para disolver $\pm 0,1 \mathrm{~g}$ de cada una de las ligninas obtenidas en los procesos, se agitó hasta que solubilizó totalmente y se agregó $1 \mathrm{ml} \mathrm{de} \mathrm{H}_{2} \mathrm{SO}_{4} 0,1 \mathrm{~N}$, homogenizando hasta obtener coloración amarilla.

Luego del análisis cualitativo se procedió a la caracterización espectroscópica del producto (lignina), usando para ello la espectroscopia UV (ultravioleta) e IR (infrarrojo), aplicándolo a los productos obtenidos de los métodos Sosa, Sulfato y Fukushina, los cuales mostraron resultados óptimos para la lignina en la extracción.

De esta manera se procedió a trabajar con el carbón tipo leonardítico procedente de la zona carbonífera del norte de Boyacá, cuya muestra fue tratada de acuerdo a la norma ASTM D2234-98 hasta obtener partículas de $\pm 0,25 \mathrm{~mm}$ (milímetros), este carbón se caracterizó mediante un análisis próximo (determinación de humedad, cenizas y materia volátil) y último (determinación de $\mathrm{N}$ y $\mathrm{S}$ ) de acuerdo a las normas ASTM D3173, 3174, 3175 y 3179-73, 3177-75 respectivamente.

Después de caracterizado el carbón leonardítico, se realizó la extracción de la lignina, para esto se extrajeron ácidos húmicos empleando $10 \mathrm{~g}$ de carbón y $250 \mathrm{ml}$ de $\mathrm{KOH} \mathrm{0,1} \mathrm{N}$, con agitación constante durante 48 h se filtró y el sólido (carbón libre de ácidos húmicos) se reservó para la posterior extracción de lignina.

La muestra de carbón libre de ácidos húmicos se trabajó aplicando el método Sosa para la extracción de lignina tomando $10 \mathrm{~g}$ de esta, $75 \mathrm{ml} \mathrm{de} \mathrm{NaOH}$ $10 \% \mathrm{P} / \mathrm{V}$ y $50 \mathrm{ml}$ de $\mathrm{Na}_{2} \mathrm{CO}_{3} 10 \% \mathrm{P} / \mathrm{V}$ en un balón de reacción, esta mezcla se dejó en reflujo hasta ebullición durante $48 \mathrm{~h}$, pasado este tiempo el producto se filtró, obteniendo así el licor con lignina como filtrado, el cual se acidificó hasta $\mathrm{pH} 2$ con $\mathrm{H}_{2} \mathrm{SO}_{4}$ y se lavó con agua deionizada hasta obtener $\mathrm{pH} 6$, teniendo de esta manera la lignina libre de impurezas que por último fue secada a $55^{\circ} \mathrm{C}$, pesada y almacenada. Esta lignina tuvo el mismo tipo de caracterización que las ligninas obtenidas en el proceso anterior.

Una vez caracterizada la lignina, se dio lugar a la preparación del producto maderable donde se utilizaron $0,5 \mathrm{~g}$ de fenol y $3 \mathrm{ml}$ de formaldehído junto con $0,4 \mathrm{~g}$ de lignina disuelta en $1 \mathrm{ml}$ de $\mathrm{NaOH} 12 \% \mathrm{P} / \mathrm{V}$. Esta mezcla se agitó en un beaker por un periodo de $1 \mathrm{~min}$ aproximadamente, luego se agregó $0,5 \mathrm{~g}$ de fibra natural (papel, aserrín, viruta, bagazo de caña o celulosa pura) previamente humedecida, se agitó y se calentó durante $25 \mathrm{~min}$ a 45 y $55^{\circ} \mathrm{C}$, posteriormente se elevó la temperatura a $80-90^{\circ} \mathrm{C}$ durante 50 min o hasta que alcanzó una dureza adecuada.

\subsection{Segunda etapa}

Con base a los resultados obtenidos en la primera etapa se planteó una segunda utilizando como materia prima la turba debido a su mayor porcentaje de 


\section{con-ciencias}

lignina, con el fin de obtener un mayor rendimiento en la extracción; la turba fue previamente caracterizada y preparada con la misma metodología utilizada para el carbón leonardítico de la primera etapa. También se modificaron las condiciones de la extracción debido a que se evidenció una contaminación con $\mathrm{SiO}_{2}$ en las ligninas obtenidas anteriormente, y se probaron otros tipos de resinas con el fin de optimizar la calidad del producto maderable.

Por otra parte, la extracción se modificó en cuanto a la disminución del tiempo y el aumento en la presión utilizando una autoclave; la extracción de lignina para turba se llevó a cabo a 115 y $134{ }^{\circ} \mathrm{C}$, $1,03 \mathrm{~atm}$, con un tiempo de 1 y $2 \mathrm{~h}$, y se siguió con la misma metodología desarrollada en la primera fase hasta obtener la lignina caracterizada.

El producto maderable se trabajó utilizando resinas previamente preparadas del tipo fenol-formaldehído, para esto se utilizaron $5 \mathrm{~g}$ de fenol, $5 \mathrm{ml}$ de formaldehído y 2 gotas de $\mathrm{H}_{2} \mathrm{SO}_{4}$, este proceso se llevó a $120^{\circ} \mathrm{C}$ en constante agitación durante 10 min y se dejó enfriar; también se utilizaron del tipo resorcinol-formaldehído para lo cual se emplearon 2 $\mathrm{g}$ de resorcinol, formaldehído y 3 gotas de glicerina y se llevó a $80{ }^{\circ} \mathrm{C}$ en constante agitación durante 10 min, y se dejó enfriar.

El producto maderable se elaboró utilizando 0,75 $\mathrm{g}$ de resorcinol, $5 \mathrm{ml}$ de formaldehído y 3 gotas de glicerina, se agitó por un período de $2 \mathrm{~min}$ aproximadamente y después se le agregó $0,15 \mathrm{~g}$ de lignina extraída, se agitó y se llevó a calentamiento durante 6 min a una temperatura entre 65 y $75^{\circ} \mathrm{C}$; transcurrido este tiempo se le añadieron $0,15 \mathrm{~g}$ de bagazo de tallo de plátano finamente molido, partículas de $0,4 \mathrm{~mm}$ y $0,75 \mathrm{~g}$ de resina obtenida a partir de la preparación de resina fenol-formaldehído, a continuación se elevó la temperatura a $80-90{ }^{\circ} \mathrm{C}$ durante $25 \mathrm{~min}$; una vez endurecido, se retiró y se desmoldó para ser sometido a corrientes de aire caliente durante $30 \mathrm{~min}$, posteriormente se dejó en reposo por un período de $48 \mathrm{~h}$.

\subsection{Tercera etapa}

En esta etapa se pretende modificar estructuralmente la lignina extraída de una muestra de turba a partir del método Sosa modificado con presión y estandarizado en la segunda etapa; para esto se van a utilizar dos tipos de métodos, el método físico (radiación UV) y el método químico $(\mathrm{NaOH})$. El primero se va a trabajar exponiendo $1 \mathrm{~g}$ de lignina extraída a la radiación emitida por una lámpara de luz ultravioleta de $4 \mathrm{~W}$ y $110 \mathrm{Vca}$, por periodos de tiempo de 12, 24, 36 y $48 \mathrm{~h}$; para el segundo método se utilizarán $2 \mathrm{~g}$ de lignina extraída disuelta en 20 $\mathrm{ml}$ de $\mathrm{NaOH}$ al $2 \% \mathrm{P} / \mathrm{V}$, esto se va a llevar a $170{ }^{\circ} \mathrm{C}$ y $1.03 \mathrm{~atm}$, por un periodo de tiempo de $90 \mathrm{~min}$ en una autoclave; después se introducirá en un baño de hielo por 10 min y se neutralizará con $\mathrm{H}_{2} \mathrm{SO}_{4}$.

Posteriormente se pretende comprobar la despolimerización a través de la reacción de Mannich, la cual consiste en preparar dos mezclas por separado, en una se mezclan $0,5 \mathrm{ml}$ de formaldehído, 2,05 $\mathrm{ml}$ de dietilamina con $12,5 \mathrm{ml}$ de dioxano, y en la otra $12,5 \mathrm{ml}$ de dioxano con $0,25 \mathrm{~g}$ de lignina despolimerizada, éstas se disolverán en un beaker con agitación constante durante $1 \mathrm{~h}$. Seguidamente, se llevarán a reflujo hasta ebullición por $3 \mathrm{~h}$ y al producto obtenido se le determinará $\mathrm{N}$ por el método Kjendahl; estos resultados serán comparados con los obtenidos para la lignina sin despolimerizar.

Con el fin de aumentar los puntos reactivos de la lignina despolimerizada se va a realizar la hidroximetilación de la lignina, la cual consistirá en tomar $4 \mathrm{ml} \mathrm{de} \mathrm{H}_{2} \mathrm{O}$ dest. con $2 \mathrm{~g}$ de lignina despolimerizada y se alcalinizará a pH 12 con $\mathrm{NaOH} 30 \%$ para después calentar a $58^{\circ} \mathrm{C}$ en reflujo, alcanzando esta temperatura se agregarán $3,3 \mathrm{ml}$ de formaldehído y se continuará el reflujo a temperatura constante durante $8 \mathrm{~h}$. Debido a lo tóxico del formaldehído, se plantea sustituir por glioxal.

Para realizar el producto maderable se van a realizar tres diferentes tipos de resinas con la lignina hidroximetilada, las cuales van a tener como base el formaldehído, esto con el fin de establecer cuál de estas presenta mejores características. 


\section{con-ciencias}

Las resinas se elaborarán con $18,8 \mathrm{~g}$ de fenol, melanina o urea para cada caso, $8 \mathrm{ml}$ de una solución de $\mathrm{MeOH} / \mathrm{H}_{2} \mathrm{O}$ 1:4, y $10 \mathrm{ml}$ de formaldehído, mezcla que se realizará a $40{ }^{\circ} \mathrm{C}$ en reflujo durante $30 \mathrm{~min}$ bajo agitación constante, luego se incorporarán 20 g de $\mathrm{NaOH}$ en 4 partes de 5 g cada $15 \mathrm{~min}$, para llevar el reflujo a $94^{\circ} \mathrm{C}$ durante $60 \mathrm{~min}$. Pasado el tiempo se va a enfriar en un baño de hielo.

La resina con las mejores características será la utilizada en la elaboración del producto maderable que consistirá en tomar $15 \mathrm{~g}$ de lignina hidroximetilada y $6,5 \mathrm{~g}$ de ésta misma, y llevarlas a $50^{\circ} \mathrm{C}$, agregando $5 \mathrm{ml}$ de pMDI y $2,5 \mathrm{ml}$ de triacetina; se continuará el calentamiento y se agregará el $50 \%$ del volumen presente en cascarilla de arroz, se continuará con el calentamiento a $50{ }^{\circ} \mathrm{C}$, moldeando el producto maderable, el cual al final se va a prensar por $30 \mathrm{~min}$ a una temperatura de $200{ }^{\circ} \mathrm{C}$ con corrientes de aire y posteriormente se desmoldará. Éste también se puede realizar con las resinas elaboradas a base de glioxal como sustituyente del formaldehído. Al producto maderable se le evaluarán las propiedades de resistencia mediante pruebas físico-químicas y mecánicas.

\section{Resultados}

Las muestras de turba y carbón leonardítico utilizadas para cada etapa fueron caracterizadas por las normas ASTM y cumplieron con las características propias para ser catalogadas como tales.

En la tabla 1 se dan a conocer los resultados de la extracción de lignina en la primera etapa, y en la tabla 2 los de la segunda.

Tabla 1. Rendimientos para los métodos de extracción Sosa, Bisulfito, Sulfato y Fukushina empleados en la extracción de lignina 1a etapa [2].

\begin{tabular}{|c|c|c|c|}
\hline $\begin{array}{c}\text { TIPO DE } \\
\text { MUESTRA }\end{array}$ & $\begin{array}{c}\text { MÉTODO DE } \\
\text { EXTRACCIÓN }\end{array}$ & $\begin{array}{l}\text { PESO LIGNINA } \\
\text { PURIFICADA (g) }\end{array}$ & $\begin{array}{c}\% \\
\text { RENDIMIENTO }\end{array}$ \\
\hline \multirow{4}{*}{ CEDRO } & $\begin{array}{c}\text { Sosa } \\
(\mathrm{NaOH} 20 \% \mathrm{P} / \mathrm{V})\end{array}$ & 2,22 & 22,2 \\
\hline & Bisulfito & \multicolumn{2}{|c|}{$* *$} \\
\hline & Sulfato & 0,70 & 7,00 \\
\hline & Fukushina & & \\
\hline \multirow{4}{*}{ PINO } & $\begin{array}{c}\text { Sosa } \\
(\mathrm{NaOH} 20 \% \mathrm{P} / \mathrm{V})\end{array}$ & 2,58 & 25,80 \\
\hline & Bisulfito & \multicolumn{2}{|c|}{$* *$} \\
\hline & Sulfato & \multicolumn{2}{|c|}{$* *$} \\
\hline & Fukushina & \multicolumn{2}{|c|}{$* *$} \\
\hline CARBÓN & $\begin{array}{c}\text { Sosa } \\
(\mathrm{NaOH} 20 \% \mathrm{P} / \mathrm{V})^{*}\end{array}$ & 0,12 & 1,20 \\
\hline
\end{tabular}

* Método Sosa estandarizado.

** Los resultados obtenidos fueron tan bajos que fue imposible pesarlos, lo que dificulto su cuantificación. 
Tabla 2. Rendimientos para el método Sosa empleado en la extracción de lignina a partir de turba 2a etapa [3].

\begin{tabular}{|c|c|c|c|c|c|c|}
\hline $\begin{array}{c}\mathrm{T} \\
\left({ }^{\circ} \mathrm{C}\right)\end{array}$ & $\begin{array}{l}\mathrm{NaOH} \\
(\mathrm{P} / \mathrm{V})\end{array}$ & $\begin{array}{l}\text { LIGNINA } \\
\text { (g) }\end{array}$ & $\begin{array}{c}\text { PORCENTAJE } \\
\text { EN MUESTRA } \\
\text { DE } 8 \mathrm{~g}\end{array}$ & $\begin{array}{c}\mathrm{NaOH} \\
(\mathrm{P} / \mathrm{V})\end{array}$ & $\begin{array}{l}\text { LIGNINA } \\
\text { (g) }\end{array}$ & $\begin{array}{c}\text { PORCENTAJE } \\
\text { EN MUESTRA } \\
\text { DE } 8 \mathrm{~g}\end{array}$ \\
\hline \multirow{4}{*}{115} & $10 \%$ & * & * & $10 \%$ & 0,53 & $6,62 \%$ \\
\hline & $20 \%$ & 1,35 & $16,87 \%$ & $20 \%$ & 1,90 & $23,75 \%$ \\
\hline & $30 \%$ & 1,10 & $13,75 \%$ & $30 \%$ & 1,16 & $14,5 \%$ \\
\hline & $40 \%$ & $*$ & * & $40 \%$ & 0,15 & $1,87 \%$ \\
\hline \multirow{5}{*}{134} & $10 \%$ & $*$ & * & $10 \%$ & 0,65 & $8,12 \%$ \\
\hline & $20 \%$ & 1,66 & $20,75 \%$ & $20 \%$ & 2,10 & $26,25 \%$ \\
\hline & $30 \%$ & 1,13 & $14,125 \%$ & $30 \%$ & 1,26 & $15,75 \%$ \\
\hline & $40 \%$ & $*$ & $*$ & $40 \%$ & 0,32 & $4,00 \%$ \\
\hline & \multicolumn{3}{|c|}{ Tiempo (1 Hora) Presión 1,03 atm } & \multicolumn{3}{|c|}{ Tiempo (2 Hora) Presión 1,03 atm } \\
\hline
\end{tabular}

* Los resultados obtenidos fueron tan bajos que fue imposible pesarlos, lo que dificulto su cuantificación.

Los porcentajes de lignina obtenidos de la madera en la primera etapa se acercan a los resultados teóricos esperados para este tipo de maderas [2], lo que indica que los métodos modificados en condiciones de laboratorio son adecuados para la optimización del proceso de extracción de lignina con otros tipos de muestras, como lo son los carbones de bajo rango, en beneficio de la elaboración de la madera sintética.

Tabla 3. Solubilidad de las ligninas obtenidas para la 1a [2] y segunda etapa [3].

\begin{tabular}{|c|c|c|c|c|}
\hline SOLVENTE & \multicolumn{2}{|c|}{ FRÍO } & \multicolumn{2}{c|}{ CALIENTE } \\
\hline & $1^{\mathrm{a}}$ Etapa & $2^{\mathrm{a}}$ Etapa & $1^{\mathrm{a}}$ Etapa & $2^{\mathrm{a}}$ Etapa \\
\hline $\mathrm{H}_{2} \mathrm{O}$ & + & + & + & + \\
\hline Metanol & - & - & - & - \\
\hline Etanol & - & - & - & - \\
\hline $\mathrm{NaOH} 10 \% \mathrm{P} / \mathrm{V}$ & + & + & + & + \\
\hline $\mathrm{H}_{2} \mathrm{SO}_{4} 98 \%$ & PS & PS & PS & PS \\
\hline $\mathrm{HCl} 10 \% \mathrm{~V} / \mathrm{V}^{\mathrm{NaHCO}} 10 \% \mathrm{P} / \mathrm{V}$ & - & - & - & - \\
\hline Éter etílico & PS & PS & PS & PS \\
\hline $\mathrm{CCl}_{4}$ & - & - & - & - \\
\hline Piridina & - & - & - & - \\
\hline
\end{tabular}

+ hace referencia a solubilidad completa en el solvente, - insoluble y PS parcialmente soluble. 


\section{con-ciencias}

De acuerdo con los resultados mostrados en la Tabla 3 , se evidencia la solubilidad de la lignina extraída en $\mathrm{NaOH}$, lo cual confirma la viabilidad del método Sosa, además la coloración oscura tomada en este solvente indica la existencia de grupos polihidroxifenólicos [13] y/o quinonas. Por otro lado, la parcial solubilidad de la lignina en solventes aromáticos nitrogenados como la piridina indica que el producto obtenido es de tipo aromático. Un aspecto para tener presente es la solubilidad en $\mathrm{NaHCO}_{3} 10 \% \mathrm{P} / \mathrm{V}$, la cual ocurre debido a las fracciones ácidas del compuesto extraído. En relación a la solubilidad presentada en el ácido sulfúrico se puede establecer que éste reacciona fácilmente con compuestos polihidroxifenólicos de alto peso molecular que son fácilmente sulfonados o que contienen más de tres anillos aromáticos.

El proceso analítico inorgánico comprendió la determinación de los principales aniones y cationes de modo cualitativo, observando resultados negativos para todos estos. En cuanto al análisis orgánico cualitativo primario se identificó principalmente un $\mathrm{pH}$ mayor de 4,8 , un resultado positivo para aromaticidad en la prueba a la llama, reducción del $\mathrm{KMnO}_{4}$ no inmediata y prueba positiva de $\mathrm{Br}_{2} / \mathrm{CCl}_{4}$, lo cual indica que el compuesto presenta insaturaciones y puede contener grupos $\mathrm{OH}$ secundarios o primarios, o de igual modo diariles.

En la aplicación de las pruebas específicas para lignina se desarrolló la prueba de anilina en $\mathrm{H}_{2} \mathrm{SO}_{4}$ [14], en la que el resultado fue positivo, ya que se obtuvo una coloración amarilla característica para el polímero. También se llevó a cabo la prueba de Wiesner, la cual denotó una baja solubilidad del compuesto, lo que dificultó el desarrollo de la coloración específica que debe desarrollar la floroglucina etanólica, ésta debe ser rojo guinda para que dicha prueba sea positiva, además debe encontrarse en un medio celulósico (lignina+celulosa), lo cual no es posible en el producto de la primera etapa ya que el carbón leonardítico no posee celulosa dentro de su estructura, por lo cual esta prueba se considera negativa para el extracto [15], en la segunda fase el color fue tenue por lo que se consideró como prueba positiva.
Es importante tener en cuenta que una de las características primordiales para que esta prueba sea positiva es la existencia de aldehídos terminales en el polímero de la lignina. Por lo tanto, podemos asegurar que la estructura de la lignina extraída en la primera etapa no posee este tipo de grupos funcionales, mientras que la estructura de la segunda sí.

Adicionalmente, al aplicarse la prueba de Maulé a la lignina extraída de la primera etapa se evidenciaron diferentes coloraciones, las cuales no son características para el polímero de la lignina, la tonalidad rojo oscuro típica que indica un resultado positivo no fue evidente en la totalidad de las mismas, en varias se apreciaron coloraciones verde claro, verde oliva, verde o café verdoso. Por el contrario, los resultados de estas pruebas en la segunda etapa fueron positivos, lo que indica la presencia de grupos siringilo propios de la lignina.

Para la espectroscopia UV de caracterización fue necesario solubilizar las ligninas extraídas en la primera y segunda etapa mediante cada uno de los métodos de extracción, en $\mathrm{NaOH} 10 \%$ P/V y $\mathrm{MeOH}$ anhidro.

La Banda I (215-218 nm (nanómetros)), es una banda intensa correspondiente a fenoles, lo cual es confirmado con una segunda banda (Banda II), la cual está presente hacia $\pm 260 \mathrm{~nm}$, correspondiente, de igual manera, a fenoles. Sin embargo, es de considerar que esta banda también puede ser debida a enlaces del grupo carbonilo $\mathrm{C}=\mathrm{O}$, que se ven desplazados a menores longitudes de onda por la polaridad del solvente que se empleó para la lectura, presentando una baja intensidad. En la mayoría de los casos se evidencia una tercera banda (Banda III) que se presenta entre 385-389 nm para la primera etapa, que puede deberse a sustituciones en el anillo aromático (pueden ser $\mathrm{OH}, \mathrm{O}-\mathrm{Met}$, etc.), y entre 207-208 nm para la segunda etapa que puede deberse a cadenas laterales [16]. Para este análisis se empleó un equipo Perkin Modelo Lamda 10, los resultados se muestran en la Tabla 4. 
Tabla 4. Principales bandas encontradas para el espectro UV de las diferentes ligninas extraídas en la primera [2] y segunda etapa [3].

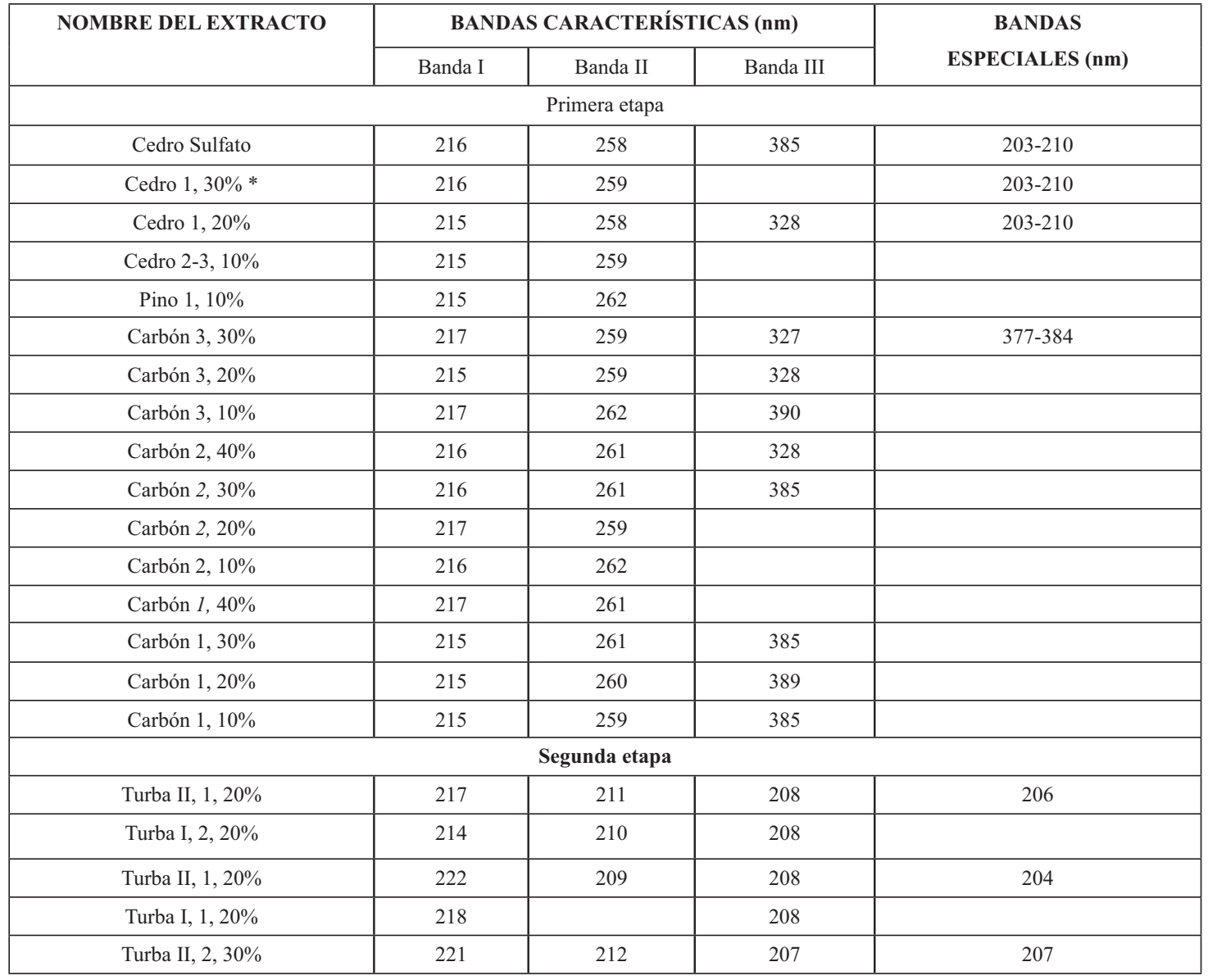

* I y II hace referencia a 115 y 134 oC; 1, 2 y 3 hace referencia al tiempo de extracción en horas; 10, 20, 30 y $40 \%$ hace referencia a la concentración de $\mathrm{NaOH}$ en $\% \mathrm{P} / \mathrm{V}$, empleado en la solución extractante.

En lo referente a la espectroscopia IR de las ligninas extraídas en las dos etapas mediante cada uno de los métodos, se encontraron tendencias bastante marcadas en las bandas encontradas para la caracterización del polímero en relación con sus grupos funcionales.

En las diferentes ligninas obtenidas por los diferentes medios de extracción se pueden encontrar tendencias generales que corresponden a los grupos $-\mathrm{OH}$, aromaticidad, grupos $\mathrm{C}=\mathrm{O}$, algunas especificaciones de acuerdo con el tipo de lignina que se está extrayendo tanto de las maderas como del carbón leonardítico y de la turba, estas interpretaciones fueron dadas de acuerdo a las referencias obtenidas [17]; [18] los resultados se muestran en la Tabla 5. 


\section{con-ciencias}

Tabla 5. Análisis de las principales bandas halladas en el espectro de IR de las diferentes ligninas extraídas en la primera [2] y segunda [3] etapa.

\begin{tabular}{|c|c|c|}
\hline MUESTRA & PRINCIPALES BANDAS $\left(\mathrm{cm}^{-1}\right)$ & INTERPRETACIÓN \\
\hline \multirow[b]{3}{*}{ Cedro $1,10 \% *$} & $3000-3700$ con pico en 3400 (alta intensidad) & Estiramiento O-H Grupo -OH \\
\hline & 3000 (con alta intensidad en la mayoría de los casos solapada) & $\mathrm{C}-\mathrm{H}$ aromático \\
\hline & 2900 (de mediana intensidad solapada) & C-H alifático \\
\hline \multirow{2}{*}{$\begin{array}{l}\text { Carbón } 1.10 \% \\
\text { Carbón 1. } 20 \% \\
\text { Carbón 1. } 30 \%\end{array}$} & 2400-2000 (múltiples bandas de baja intensidad) & Bandas atribuidas a aromaticidad \\
\hline & $1800-2000$ (dos bandas de baja intensidad) & Aromaticidad \\
\hline \multirow{2}{*}{$\begin{array}{l}\text { Carbón 2. } 10 \% \\
\text { Carbón 2. } 30 \% \\
\text { Carbón 2. } 40 \%\end{array}$} & $\begin{array}{c}1650-1625 \text { (mediana intensidad) } \\
1400-1390 \text { (alta intensidad) }\end{array}$ & $\mathrm{C}=\mathrm{O}$ conjugado \\
\hline & $1250-1230$ (alta intensidad) & ácido p-cumarílico \\
\hline $\begin{array}{l}\text { Carbón } 3.20 \% \\
\text { Carbón } 3.30 \%\end{array}$ & $1118-1025$ (alta intensidad) & C-C anillos aromáticos \\
\hline \multirow{2}{*}{$\begin{array}{l}\text { Turba II, } 2,20 \% \\
\text { Turba I, } 2,20 \% \\
\text { Turba II, } 1,20 \%\end{array}$} & 948-937 (mediana intensidad) & Anillo siringilico \\
\hline & $\begin{array}{c}\text { 8010-808 (mediana intensidad) } \\
\text { Aprox. } 400\end{array}$ & $\begin{array}{l}\text { C-O alcoholes secundarios } \\
\text { Guayacílico fenólico } \\
\text { Unidades sinapílico y } \\
\text { p-cumarílico } \\
\text { C-H aromático } \\
\text { Indeterminada }\end{array}$ \\
\hline
\end{tabular}

* I y II hace referencia a 115 y 134 oC; 1, 2 y 3 hacen referencia al tiempo de extracción en horas; 10, 20, 30 y $40 \%$ hacen referencia a la concentración de $\mathrm{NaOH}$ en $\% \mathrm{P} / \mathrm{V}$, empleado en la solución extractante.

Con el fin de determinar el tipo de contaminante en la lignina extraída del carbón leonardítico de la primera etapa se realizó la determinación del porcentaje de cenizas, el cual constituye la porción de material inorgánico que puede estar incluido en las diferentes sustancias. Este procedimiento se llevó a cabo a $750{ }^{\circ} \mathrm{C}$, empleando $1 \mathrm{~g}$ de cada una de las ligninas obtenidas. De esta manera, se evidenció que el extracto presentaba un significativo porcentaje de materia inorgánica (promedio $23.44 \%$ ), lo que obligó a determinar qué tipo de materia estaba alterando la composición de ésta, a través de reacciones químicas [19] se encontró que el agente contaminante prevalente en el producto de extracción por medios básicos era el Silicio como $\mathrm{SiO}_{2}$. Después de este análisis se determinó el efecto que causaba el $\mathrm{SiO}_{2}$ sobre las bandas encontradas en caracterización espectroscópica para la lignina, encontrando bandas de mediana intensidad hacia $500 \mathrm{~cm}^{-1}$ que correspondían al silicio. 


\section{con-ciencias}

El polímero de la lignina fue tratado con formaldehído y fenol en la generación de la resina fenolformaldehído, que posteriormente fue mezclada con papel, viruta y bagazo de caña con el fin de darle más volumen y consistencia al producto maderable en la primera etapa; en la segunda, el producto maderable se trabajó utilizando resinas resorcinolformaldehído con una mezcla también de resinas fenol-formaldehído y como fibra natural se utilizó bagazo de tallo de plátano con el mismo fin con el que se había utilizado la fibra natural en la primera etapa. Con este tratamiento se obtuvo un producto con características de dureza apreciables, color café claro y densidades entre 1.1 y $1.5 \mathrm{~g} / \mathrm{ml}$, mostrando así que la reacción de polimerización era viable empleando el producto extraído, ya que éste actuaba como aglomerante de las diferentes fibras naturales utilizadas. Este agente aglomerante en combinación con aserrín y papel generó un producto con mayor resistencia, en comparación con el producto obtenido con el bagazo de caña en la primera etapa.

El proceso de formación del producto maderable se da debido a que la estructura de la lignina permite que se dé un proceso de polimerización acelerado que reemplaza al fenol y, al mismo tiempo, genera diferentes enlaces que dan como resultado una estructura polimérica no convencional (no definida) que dota a la resina de una dureza notable.

Un posible modo de reacción para la lignina en la resina fenol-formaldehído F-F de la primera y segunda etapa (ver Figura 3) es la formación de la resina F-F por condensación, en la cual la parte de los hidroxilos del aromático presentes en la lignina interaccionan de igual modo por condensación con

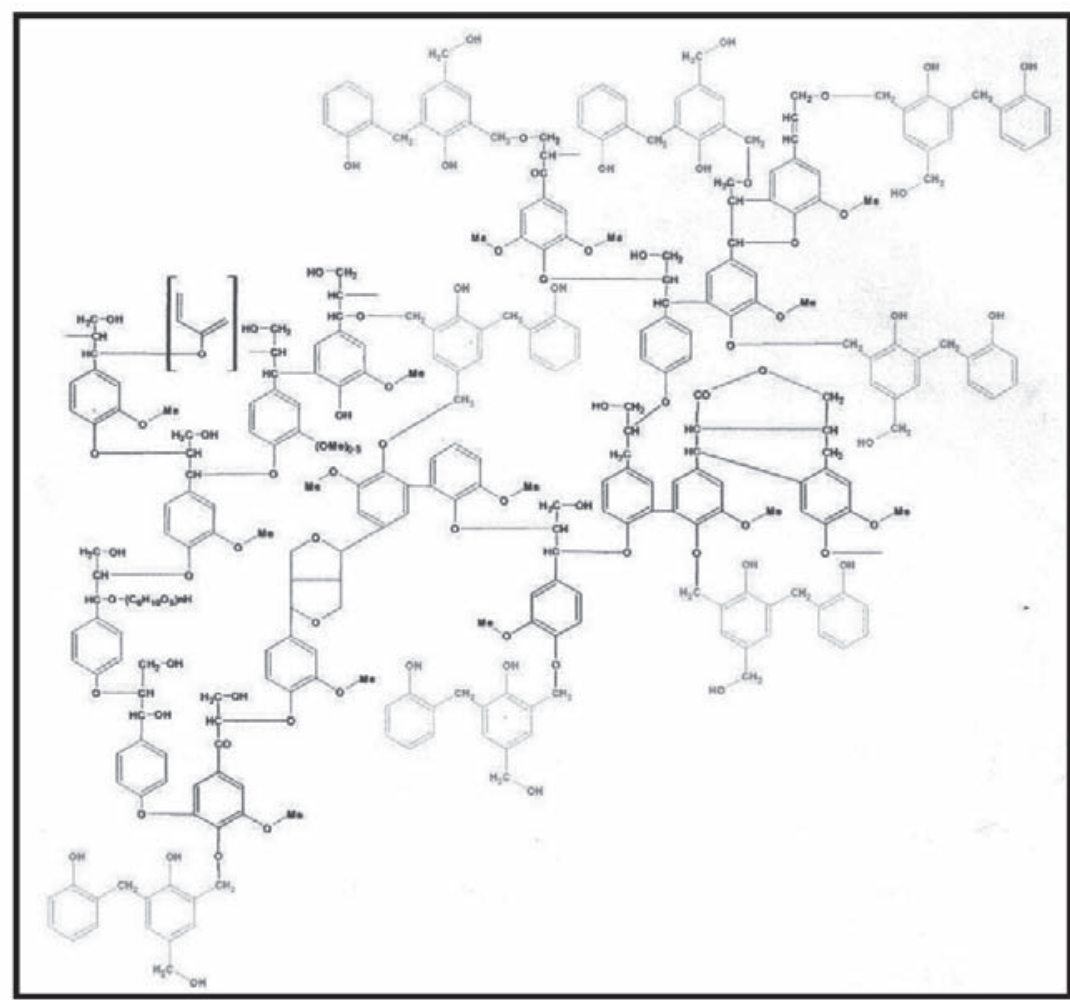

Figura 3. Posibles uniones de la resina F-F a la estructura de la lignina para formar resinas L-F-F. (La estructura mostrada en negro constituye la posible estructura de la lignina y las estructuras aromáticas en gris son las uniones de la resina F-F). [2]. 


\section{con-ciencias}

la resina, generando la unión lignina-fenol-formaldehído L-F-F, por medio de un enlace tipo éter. Sin embargo, de acuerdo con la estructura de la lignina [2], ésta presenta diferentes grupos hidroxílicos en diversas partes, lo que dificulta que la resina F-F interaccione de un único modo, ésta lo hace de modo aleatorio generando una estructura poco determinable en relación con los polímeros que en su mayoría presentan unidades que se repiten de acuerdo a su condensación.

La fibra natural para la formación del producto maderable en las diferentes etapas de la investigación no reacciona directamente con la resina L-F-F sino que se mezcla con ésta para otorgarle al producto maderable más cuerpo y consistencia (ver Figura 4).

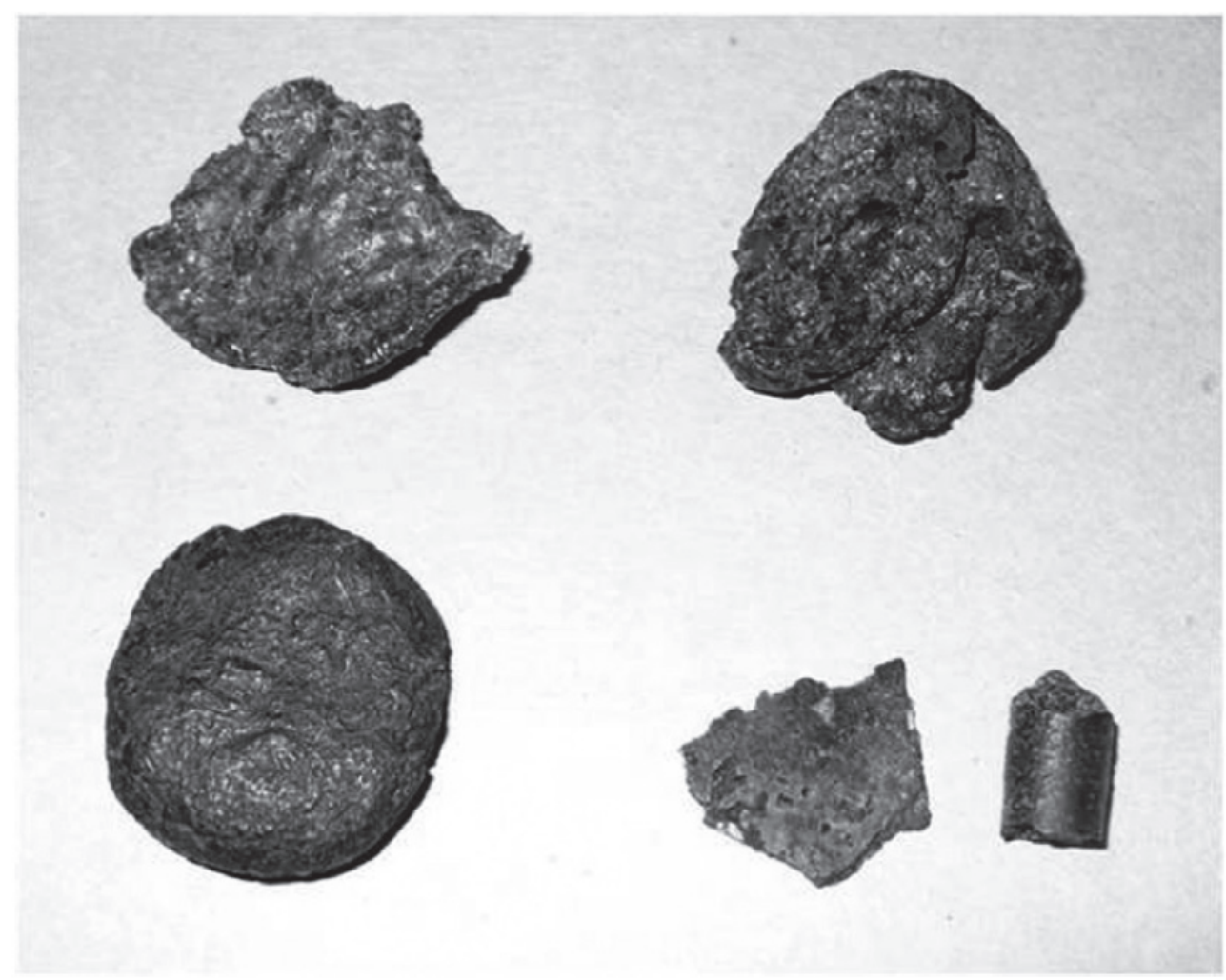

Figura 4. Productos maderables obtenidos en cada una de las etapas. Primera etapa (arriba) y segunda etapa (abajo).

Los resultados de la tercera etapa no se muestran debido a que está en proceso de desarrollo actualmente.

\section{Conclusiones}

La turba y el carbón de bajo rango por su composición química y por su proceso de carbonificación son excelentes fuentes de lignina, por lo que pueden ser útiles en la industria maderera.

Debido a la alta solubilidad que presenta la lignina en $\mathrm{NaOH}$, es posible que éste sea el principal agente que compone la mezcla extractante en el proceso de extracción de lignina. 


\section{con-ciencias}

Las resinas utilizadas en la elaboración del producto maderable aumentan sus características físicas y químicas cuando se mezclan con la lignina extraída de la turba y del carbón de bajo rango.

El silicio que se encuentra en la lignina como impureza en la primera etapa no afecta la polimerización en la generación de la resina lignina-fenol-formaldehído, ni en la elaboración del producto maderable.

El uso de la lignina extraída en la formación de resinas tipo lignina-fenol-formaldehído es viable ya que entre éstos se da un proceso de polimerización, permitiéndoles actuar como aglomerante de fibras naturales.

\section{Financiamiento}

El financiamiento ha sido por parte de la Universidad Distrital Francisco José de Caldas y de sus autores.

\section{Agradecimientos}

A Dios y a la Línea de Investigación en Carbones de la Universidad Distrital Francisco José de Caldas.

A Sandra Patricia Gaitán M., Luis Fernando Ruge F. y Camilo Andrés Mahecha M. por su participación activa en el desarrollo de la primera etapa de la investigación; también a Margarita Téllez Marín y Leyan Milena Torres G., por su aporte en la realización de la segunda etapa.

\section{Referencias bibliográficas}

[1] R. Shreve, Manual de Procesos Químicos en la Industria. México: Mc Graw Hill, pp. 726-728, 1992.

[2] S. Gaitán, L. Ruge, C. Mahecha, "Estudio preliminar para la extracción de lignina a partir del carbón leonardítico y su posterior empleo en generación de un producto maderable", Trabajo de grado para optar al título de Licenciado en Química UDFJC, Bogotá, 2006, pp. 15-115.

[3] M. Téllez, L. Torres, "Estandarización del método de extracción de lignina contenida en turba y obtención de una resina polimérica a partir de este producto", Trabajo de grado para optar al título de Licenciado en Química UDFJC, Bogotá, 2009, pp. 11-87.
[4] N. El Mansouri, "Despolimerización de lignina para su aprovechamiento en adhesivos para producir tableros de partículas", Trabajo de grado para optar al título de Doctor en Ingeniería Química y Procesos, Universidad de Tarragona, España, 2006, pp. 1-141.

[5] Ecogaia, La deforestación disminuye en el mundo, pero continúa a ritmo alarmante en muchos países 2010, [en línea]. Disponible en: www.ecogaia.com

[6] D. Eklund y T. Lindström, "Chapter II: The fibre and its behaviour during papermaking". Finland, En: Eklund, D. y Lindström, T.(Eds.). Paper Chemistry. An Introduction. DT Paper Science Publications Grankulla, pp. 8-52., 1991.

[7] H. García, J. Martín, J. Rodríguez, "Posi- 


\section{con-ciencias}

bilidades de aprovechamiento de la lignina en la industria química", Ingeniería Química, pp. 249-254, octubre 1984.

[8] J. Martínez, C. Gómez, D. Restrepo, P. Gañán, "Evaluación del curado de resinas fenólicas tipo novolaca modificadas con lignina por espectroscopia infrarroja por transformada de Fourier", Suplemento de la Revista Latinoamericana de Metalurgia y Materiales, S1, no. 3, pp. 1173-1179, 2009.

[9] J. Martínez, J. Velásquez, W. Ramírez, P. Gañán, "Modificación de resinas fenólicas con lignina procedente del pulpeo alcalino del bagazo de caña", Scientia et Technica Universidad de Pereira Tecnológica, vol. 13, no. 36, septiembre 2007.

[10] J. Velásquez, Utilización de lignina de licor negro para la sustitución de resinas en aglomerados de madera, Medellín Colombia: Facultad de Ingeniería Química, Grupo de investigación Pulpa y Papel, UPB, 2007.

[11] El Carbón En La Vida Diaria, El carbón mineral, [en línea]. Disponible en: www. oviedocorreo.es/personales/carbon/carbon mineral/carbon $\% 20$ mineral.ht

[12] Arquicity.com, Aglomerante, [en línea]. [Consultado: 28 de Octubre 2010]. Disponible en: www.arquicity.com/que-es-aglomerante. html

[13] J. Martínez, Análisis Orgánico Cualitativo. Bogotá: Universidad Nacional de Colombia,
1972.

[14] P. Barrer, Tratado de química orgánica. Barcelona: Manuel Marín Editor, 1944.

[15] J. Speight, The chemestry and technology of coal, segunda edición, New York: Marcell Deckker, 1994.

[16] R. Silverstein, G. Bassler, T. Morrill, Spectrometric Identification of Organic Compounds, New York: John Wiley, 1991.

[17] H. Hergert, "Infrared Spectra", en Lignins Ocurrence, Formation, Structure and Reactions, Sarkanen, K.V, Ludwing, C.H., edits., Willey-Interscience, pp. 267-293, 1971.

[18] O. Faix, Investigation of Lignin Polymer Models (DHP'S) by FTIR Spectroscopy, Holzforschung, pp. 40, 273-280, 1986.

[19] R. Brunday, Análisis Cualitativo, México: Editorial continental S.A, 1971. 